\title{
Phenobarbital pharmacokinetics in neonates and infants during extracorporeal membrane oxygenation
}

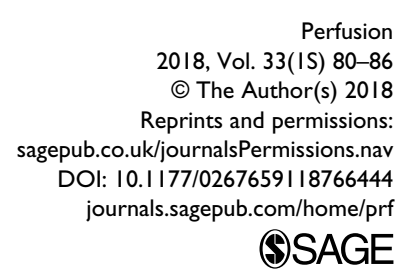

20I8, Vol. 33(IS) 80-86

(C) The Author(s) 2018 gepub.co.uk/journalsPermissions.nav DOI: 10.1 I 77/0267659| I8766444 @SAGE

\author{
Pavla Pokorná, 1,2,3 (D) Martin Šíma,2 (D) Václav Vobruba,' \\ Dick Tibboel ${ }^{1,3}$ and Ondřej Slanař
}

\begin{abstract}
Introduction: The disposition of drugs is potentially changed due to extracorporeal membrane oxygenation (ECMO) in neonates and infants.

Methods: The aim of the study was to evaluate the individual pharmacokinetics (PK) of phenobarbital and the effect of PK covariates in neonates and infants undergoing ECMO. Sixteen patients ( 7 neonates, 9 infants) treated with phenobarbital during ECMO (centrifugal-flow pump circuits) were enrolled in the PK study. Phenobarbital serum concentrations were measured using a fluorescence polarization immunoassay. Individual PK parameters - volume of distribution (Vd) and clearance $(\mathrm{CL})$ were calculated in a one-compartmental pharmacokinetic model.

Results: The mean (SD) Vd and CL values in neonates were $0.46(0.24) \mathrm{L} / \mathrm{kg}$ and $8.0(4.5) \mathrm{mL} / \mathrm{h} / \mathrm{kg}$, respectively. Respective values in infants were $0.56(0.23) \mathrm{L} / \mathrm{kg}$ and $8.5(3.1) \mathrm{mL} / \mathrm{h} / \mathrm{kg}$. PK parameters in neonates and infants were not significantly different. We observed high inter-individual variability in PK parameters (coefficients of variation [CV] were 52\% and 53\% for $\mathrm{CL}$ and $\mathrm{Vd}$, respectively). Doses were adjusted based on therapeutic drug monitoring (TDM) in $87.5 \%$ patients. Only $50 \%$ of the first measured phenobarbital serum concentrations in each patient were within the therapeutic range of $10-40$ $\mathrm{mg} / \mathrm{L}$, in comparison with $88.6 \%$ concentration measured after TDM implementation. Linear regression models showed that both $\mathrm{Vd}$ and $\mathrm{CL}$ are significantly related with body weight (BW) and length. Median optimal phenobarbital loading dose (LD) and maintenance dose (MD), calculated from pharmacokinetic data, were $15 \mathrm{mg} / \mathrm{kg}$ and $4 \mathrm{mg} / \mathrm{kg} /$ day, respectively.

Conclusions: Body weight was shown to be the main PK covariate of phenobarbital disposition. Subsequent dosing nomograms are provided for phenobarbital dosing during ECMO.
\end{abstract}

\section{Keywords}

ECMO; infants; pharmacokinetics; pharmacotherapy; phenobarbital

\section{Introduction}

Extracorporeal membrane oxygenation (ECMO) is a complex highly specialised treatment modality used in neonates and infants with reversible circulatory and/or respiratory failure. Rapidly changed drug disposition on ECMO leads to changes in drug serum concentrations (e.g. antibiotics, anticonvulsive drugs, analgosedatives) that alter the therapeutic efficacy; ${ }^{1,2}$ ECMO increases drug volume of distribution (Vd) due to priming volume, drug binding in the oxygenator and changes in albumin concentrations. ECMO is believed to change clearance (CL) of most drugs - lipophilic drugs in particular, due to sequestration in the ECMO circuits. ${ }^{3,4}$ Moreover, other factors may play a role in drug specificity - as reported for lipophilic drugs with a high hepatic $\mathrm{CL}$ (i.e. fentanyl). Higher lipophilicity of the drug $(\log P)$ results in increased Vd during ECMO., ${ }^{3,4}$ For other drugs or low hepatic and kidney CL drugs, the alteration of PK during ECMO is unknown (i.e. sufentanil, clonidine,

\footnotetext{
'Department of Pediatrics - PICU/NICU, General University Hospital, Ist Faculty of Medicine Charles University, Prague 2, Czech Republic 2Department of Pharmacology, General University Hospital, Ist Faculty of Medicine Charles University, Prague 2, Czech Republic ${ }^{3}$ Intensive Care and Department of Pediatric Surgery, Erasmus MC Sophia Childrens Hospital, Rotterdam, the Netherlands
}

Corresponding author:

Pokorna Pavla, Department of Pediatrics - PICU/NICU, General University Hospital, Ist Faculty of Medicine Charles University, Ke Karlovu 2, 12802 Prague 2, Czech Republic.

Email: pokorna.pavla@vfn.cz 
phenobarbital). However, in vitro data reported that mechanisms behind changed CL are not clear yet - it depends on drug lipophilicity, but also on the binding site saturation..$^{5-8}$

Phenobarbital, phenytoin and fos-phenytoin are anticonvulsive drugs used in neonates and infants undergoing ECMO to treat seizures (18-20\%) and withdrawal symptoms. ${ }^{8-10}$ There is insufficient data on the potential impact of ECMO on the PK of phenobarbital. ${ }^{11}$ A number of publications reported serum concentrations of phenobarbital after the loading dose (LD) to be decreased ( 24 hours) due to the large Vd, while concentrations measured during the maintenance dose (MD) regimen were still within the therapeutic range and the calculated $t_{1 / 2}$ was similar to neonates receiving phenobarbital without ECMO., 911,12 PK variability is high in this population, therefore, individual therapeutic drug monitoring (TDM) is essential as the basis for individualized dosing in critically ill neonates and infants treated with different treatment modalities. ${ }^{13,14}$ Changes in $\mathrm{Vd}$ or CL may have as a consequence that the normalized dose (i.e. dose of drug per kg) is different in ECMO neonates and children compared to non-ECMO patients. ${ }^{3}$

The aim of the study was to evaluate the PK of phenobarbital in neonates and infants during ECMO and the potential effects of covariates on phenobarbital concentrations and, subsequently, dosing.

\section{Materials and methods}

\section{Design and Setting Population}

An observational pharmacokinetic study was conducted in neonates and infants admitted to the Neonatal Intensive Care Unit of the General University Hospital in Prague and concomitantly treated with intravenous phenobarbital and ECMO. Different ECMO devices were used: Bio-Medicus or Medtronic VA ECMO, Avalon or Origen VV ECMO coating cannulas, Levitronix ${ }^{\oplus}$ CentriMag $^{\oplus}$ or Maquet Rotaflow centrifugal-flow pumps and Maquet pediatric Quadrox iD, Eurosets Pedi/Newborn or Medos Newborn oxygenators between October 2010 and January 2018.1,8 Patients meeting the following criteria were included: 1) not receiving dialysis; 2 ) having at least two phenobarbital levels measured in the course of the ECMO period. Approval of the study was provided by the Ethics Committee of the Department of Ethics, General University Hospital in Prague under the RV-project 64-165/2012. Since the study collected data retrospectively and our study involved only analysis of routine clinical data and at admission to the hospital the patients' parents signed an approved general informed consent (as a part of the standardized treatment protocol) wherein they state, inter alia, that anonymous data can be used for research, study specific informed consent was unnecessary. Exclusion criteria were severe congenital abnormalities, intracranial hemorrhage and severe bleeding due to disseminated intravascular coagulopathy (DIC).

\section{Phenobarbital dosing}

Phenobarbital (Phenobarbitalum Natricum), Desitin Arzneimitttel GmbH, Hamburg, Germany) was used as follows: an intravenous (iv) loading dose of phenobarbital was administered in 15 minutes; another LD could be given 15 minutes after the first LD if clinically indicated, until a maximum total $\mathrm{LD}$ of $40 \mathrm{mg} / \mathrm{kg}$ was reached. ${ }^{15-18}$

The maintenance dose of phenobarbital (MD) was administered in 15 minutes, divided in two to three doses every 8-12 $h,{ }^{19}$ repeated doses were given based on clinical and/or an elelctrocephalograph (EEG) response to achieve the therapeutic range of $10-40 \mathrm{mg} / \mathrm{L}$ in neonates and, based on clinical observations, in infants (in the case of withdrawal) using the Sophia Observation withdrawal Symptoms-scale (SOS)/or the Neonatal abstinence syndrome (NAS) scores. ${ }^{20,21}$

\section{Data collection}

Gender, age, length in neonates, (height in infants) and body weight, serum creatinine and phenobarbital serum concentrations (sampling times included), phenobarbital dosing and administration times were recorded in each patient. Serum creatinine levels were measured using the Jaffe photometric method without deproteinization on a Modular analyzer (Roche Diagnostics, Basel, Switzerland), while phenobarbital serum concentrations, using sparse data collections (recorded times), were measured using a fluorescence polarization immunoassay on an AxSYM analyser (Abbott Laboratories, Abbott Park, IL, USA).

\section{Pharmacokinetic analysis}

Individual PK parameters - volume of distribution, clearance and elimination half-life $\left(t_{1 / 2}\right)$ were calculated in a one-compartmental PK model with firstorder elimination kinetics based on demographic and clinical data and measured phenobarbital serum levels using $\mathrm{MWPharm}^{++}$software (MediWare, Prague, Czech Republic). The phenobarbital population PK model was individualized to maximize fitting of the simulated pharmacokinetic profile curve with observed concentration points in each patient. The fitting was performed using the Marquardt nonlinear least-square method. 
Table 1. Study population.

\begin{tabular}{llc}
\hline Neonates $(\mathrm{n}=7)$ & Median & IQR \\
\hline Body weight $(\mathrm{kg})$ & 3.5 & $3.0-3.9$ \\
Height $(\mathrm{cm})$ & 49 & $48-5 \mathrm{I}$ \\
Screat $(\mu \mathrm{mol} / \mathrm{L})$ & 58 & $41-89$ \\
ECMO duration (days) & 6 & $6-10$ \\
\hline Infants $(\mathrm{n}=9)$ & Median & IQR \\
\hline Body weight $(\mathrm{kg})$ & 8.0 & $6.1-13.0$ \\
Height $(\mathrm{cm})$ & 77 & $64-90$ \\
Screat $(\mu \mathrm{mol} / \mathrm{L})$ & 28 & $23-34$ \\
ECMO duration (days) & 16 & $10-19$ \\
\hline All patients $(\mathrm{n}=\mathrm{I6})$ & Median & $1 \mathrm{QR}$ \\
\hline Body weight $(\mathrm{kg})$ & 5.2 & $3.5-8.0$ \\
Height $(\mathrm{cm})$ & 60 & $50-77$ \\
Screat $(\mu \mathrm{mol} / \mathrm{L})$ & 33 & $25-54$ \\
ECMO duration (days) & $1 \mathrm{6}$ & $6-16$ \\
\hline
\end{tabular}

Screat: serum creatinine; ECMO: extracorporeal membrane oxygenation.

\section{Optimal loading and maintenance dose calculation}

Optimal loading doses were calculated for each patient based on individual values of $\mathrm{Vd}$, using the following formula: $\mathrm{LD}(\mathrm{mg} / \mathrm{kg})=\mathrm{Vd}(\mathrm{L} / \mathrm{kg}) \times \mathrm{C}_{\text {peak }}(\mathrm{mg} / \mathrm{L})$, where $\mathrm{C}_{\text {peak }}$ is $30 \mathrm{mg} / \mathrm{L}$ as an optimal phenobarbital peak concentration.

Optimal daily maintenance doses were calculated for each patient based on individual values of CL, using the following formula: $\mathrm{MD}(\mathrm{mg} / \mathrm{kg} /$ day $)=\mathrm{CL}(\mathrm{L} / \mathrm{h} / \mathrm{kg}) \times \mathrm{C}_{\mathrm{ss}}$ $(\mathrm{mg} / \mathrm{L}) \times 24(\mathrm{~h})$, where $\mathrm{C}_{\mathrm{ss}}$ is $25 \mathrm{mg} / \mathrm{L}$ as a midpoint of the target therapeutic range $(10-40 \mathrm{mg} / \mathrm{L})$.

\section{Statistical analysis}

Descriptive parameters means, standard deviations (SD), medians and interquartile range (IQR) were calculated using MS Excel 2010 (Microsoft Corporation, Redmond, WA, USA). The $95 \%$ confidence intervals (CI) for medians were calculated by the Bonett $\&$ Price method. ${ }^{22}$

An unpaired t-test was used to compare phenobarbital pharmacokinetic parameters (Vd and CL), normalized per $\mathrm{kg}$ of body weight, in neonates and infants.

Linear regression models were used to evaluate the relationships of phenobarbital primary pharmacokinetic parameters (Vd, CL) and the patients' demographic/clinical features (BW, height, serum creatinine, ECMO duration) using GraphPad Prism 3.02 (GraphPad Software, Inc., La Jolla, CA, USA).

\section{Results}

Sixteen patients treated with ECMO and, if needed, with phenobarbital (seizures, sedation and withdrawal syndrome) were enrolled in the PK study. Seven of them were neonates ( 4 males, 3 females), while nine were infants ( 3 males, 6 females). Demographics and clinical characteristics of the patients are summarized in Table 1. In total, $75 \%$ of the patients received a phenobarbital loading dose which ranged from 6 to $27 \mathrm{mg} / \mathrm{kg}$. The daily maintenance doses ranged between 2 and $23 \mathrm{mg} /$ $\mathrm{kg} /$ day. There were no differences in the weight-normalized MD administered to neonates and to infants (2-23 and $2-22 \mathrm{mg} / \mathrm{kg} /$ day, respectively). In $87.5 \%$ of the patients the MD were adjusted based on TDM in the course of therapy.

In total, 86 phenobarbital serum levels were obtained as sparse sampling collections while the times of sampling were precisely documented, with an average number of samples of 5.4 (2-13 concentration points per patient). The proportion of patients with measured phenobarbital serum concentrations, after LD and during $\mathrm{MD}$, in/under/above the therapeutic range is summarized in Table 2 (A). Pharmacokinetic analysis is summarized in Table 3. Phenobarbital PK parameters normalized per kg of body weight in neonates and infants were not significantly different. We observed high interindividual variability of PK parameters normalized per $\mathrm{kg}$ of body weight, demonstrated, by a coefficient of variation of $52 \%$ and $53 \%$ for CL and Vd, respectively.

Linear regression models showed that both $\mathrm{Vd}(\mathrm{L})$ and $\mathrm{CL}(\mathrm{mL} / \mathrm{h})$ are significantly related with body weight and height in infants and length in the whole study group, respectively (Figure 1). In contrast, no significant relationships were observed in neonates in weight-normalized PK parameters between CL $(\mathrm{mL} / \mathrm{h})$ and serum creatinine levels.

Median optimal phenobarbital LD and MD, simulated based on observed PK data, were 15 (95\% CI: 9.919.8) $\mathrm{mg} / \mathrm{kg}$ and $4(95 \% \mathrm{CI}: 3.5-5.4) \mathrm{mg} / \mathrm{kg} /$ day, respectively. Subsequently, we simulated the administration of this recommended dosing on the model with each individual's PK data. The proportion of patients with simulated phenobarbital serum concentration after $\mathrm{LD}$ of $15 \mathrm{mg} / \mathrm{kg}$ and during MD of $4 \mathrm{mg} / \mathrm{kg} /$ day is summarized in Table 2 (B).

\section{Discussion}

Our study demonstrated that body size was the main covariate of phenobarbital PK in neonates and infants undergoing ECMO and that therapeutic drug monitoring optimized the treatment with phenobarbital to achieve therapeutic ranges. We used the recommended target therapeutic range of phenobarbital (10-40 mg/L) in this study, however, the target therapeutic range is still not clearly established as the initial studies reported an optimal therapeutic range of phenobarbital between 15 to $30 \mathrm{mg} / \mathrm{L}^{23}$ while population PK studies recommend phenobarbital serum concentration $<50 \mathrm{mg} / \mathrm{L} .{ }^{14}$ 
Table 2. (A) Proportion of patients, N (\%), with measured phenobarbital serum concentrations after the administered loading (LD) and maintenance doses (MD) in $(10-40 \mathrm{mg} / \mathrm{L})$, under $(<10 \mathrm{mg} / \mathrm{L})$ and above $(>40 \mathrm{mg} / \mathrm{L})$ the therapeutic range. (B) Proportion of patients, $\mathrm{N}(\%)$, with simulated phenobarbital serum concentrations after simulated administration of optimal LD (I5 mg/kg) and MD $(4 \mathrm{mg} / \mathrm{kg} /$ day $)$ in $(10-40 \mathrm{mg} / \mathrm{L})$, under $(<10 \mathrm{mg} / \mathrm{L})$ and above $(>40 \mathrm{mg} / \mathrm{L})$ the therapeutic range.

\begin{tabular}{|c|c|c|c|c|}
\hline \multicolumn{2}{|c|}{ Phenobarbital serum concentration } & \multirow{2}{*}{$\frac{10-40 \mathrm{mg} / \mathrm{L}}{8(50.0)}$} & \multirow{2}{*}{$\frac{<10 \mathrm{mg} / \mathrm{L}}{6(37.5)}$} & \multirow{2}{*}{$\frac{>40 \mathrm{mg} / \mathrm{l}}{2(12.5)}$} \\
\hline (A) Measured & LD (6-27 mg/kg) & & & \\
\hline & MD (2-23 mg/kg/day) & $10(62.5)$ & $0(0.0)$ & $6(37.5)$ \\
\hline \multirow[t]{2}{*}{ (B) Simulated } & LD (15 mg/kg) & II (68.7) & $0(0.0)$ & $5(3 \mid .2)$ \\
\hline & MD (4 mg/kg/day) & 14 (87.5) & $0(0.0)$ & $2(12.5)$ \\
\hline
\end{tabular}

Table 3. Pharmacokinetic data.

\begin{tabular}{lcccc}
\hline Neonates $(\mathrm{n}=7)$ & Mean & $\mathrm{SD}$ & Median & $\mathrm{IQR}$ \\
\hline $\mathrm{Vd}(\mathrm{L})$ & $\mathrm{I} .60$ & 0.98 & $\mathrm{I} .20$ & $0.99-2.3 \mathrm{I}$ \\
$\mathrm{Vd}(\mathrm{L} / \mathrm{kg})$ & 0.46 & 0.24 & 0.46 & $0.32-0.64$ \\
$\mathrm{CL}(\mathrm{mL} / \mathrm{h})$ & 26.4 & 12.9 & 20.0 & $17.4-33.0$ \\
$\mathrm{CL}(\mathrm{mL} / \mathrm{h} / \mathrm{kg})$ & 8.0 & 4.5 & 6.5 & $5.2-9.3$ \\
$\mathrm{t}_{\mathrm{I} / 2}(\mathrm{~h})$ & 46.1 & 27.7 & 48.0 & $30.7-5 \mathrm{I} .7$ \\
\hline Infants $(\mathrm{n}=9)$ & Mean & $\mathrm{SD}$ & Median & $\mathrm{IQR}$ \\
\hline $\mathrm{Vd}(\mathrm{L})$ & 5.53 & 3.54 & 4.10 & $3.20-7.94$ \\
$\mathrm{Vd}(\mathrm{L} / \mathrm{kg})$ & 0.56 & 0.23 & 0.58 & $0.40-0.80$ \\
$\mathrm{CL}(\mathrm{mL} / \mathrm{h})$ & 80.9 & 45.2 & 68.4 & $48.0-108.5$ \\
$\mathrm{CL}(\mathrm{mL} / \mathrm{h} / \mathrm{kg})$ & 8.5 & 3.1 & 8.1 & $6.0-9.8$ \\
$\mathrm{t}_{\mathrm{I} / 2}(\mathrm{~h})$ & 47.5 & 18.0 & 44.4 & $31.6-66.5$ \\
\hline All patients $(\mathrm{n}=\mathrm{I6})$ & Mean & $\mathrm{SD}$ & Median & $\mathrm{IQR}$ \\
\hline $\mathrm{Vd}(\mathrm{L})$ & $3.8 \mathrm{I}$ & 3.33 & $3.0 \mathrm{I}$ & $\mathrm{I}, 20-4,36$ \\
$\mathrm{Vd}(\mathrm{L} / \mathrm{kg})$ & 0.49 & 0.26 & 0.46 & $0.34-0.75$ \\
$\mathrm{CL}(\mathrm{mL} / \mathrm{h})$ & 57.0 & 44.0 & 44.9 & $22.4-71.3$ \\
$\mathrm{CL}(\mathrm{mL} / \mathrm{h} / \mathrm{kg})$ & 7.8 & 4.1 & 6.8 & $5.4-9.8$ \\
$\mathrm{t}_{\mathrm{I} / 2}(\mathrm{~h})$ & 46.9 & 21.9 & 46.2 & $31.5-57.5$ \\
\hline
\end{tabular}

Vd: volume of distribution; $C L$ : clearance; $\mathrm{t}_{1 / 2}$ : elimination half-life.

We found similar high inter-individual PK variability for Vd and CL and no statistical differences in Vd or CL between neonates and infants. Vd was not as large as reported, while CL was found similar. ${ }^{12}$ We speculate that phenobarbital lipophilic/hydrophilic characteristics $(\log p=1.47)$, in particular, may play a role in the phenobarbital disposition under ECMO, potentially leading to different distribution in comparison with ECMO-induced changes in drug distribution for typical lipophilic drugs, such as analgosedatives (the mean drug loss at 24 hours reported for morphine $51 \%$, midazolam $40 \%$, fentanyl $84 \%$, sufentanil $83 \%) .{ }^{8}$ Recently, Raffaeli and co-authors performed an in vitro analysis of drugs, as reported, using the same system (hollow-fibre ECMO system), but no data on phenobarbital were reported. ${ }^{8}$ A higher LD for some lipophilic drugs may be used to achieve the target therapeutic range due to drug loss in different ECMO circuits. According to our knowledge, phenobarbital drug loss due to ECMO circuit absorption in a centrifugal pump circuit was less than predicted as only $12.5 \%$ phenobarbital serum concentrations were lower than the target range, as Vd was lower and CL was found almost similar to the reported data. ${ }^{11,24}$

In routine clinical practice, phenobarbital dosing is commonly based on body weight. In accordance, we observed strong relationships between $\mathrm{Vd}(\mathrm{L})$ and body weight and between CL $(\mathrm{mL} / \mathrm{h})$ and body weight (Figure 1). Based on these relationships, we propose a dosing nomogram for both loading and daily maintenance doses (Figure 2).

In agreement with previously published studies, we also observed a relatively higher proportion of patients (37.5\%) with subtherapeutic levels after the start of phenobarbital treatment. ${ }^{12,25}$

We had to use relatively higher MD (to $23 \mathrm{mg} / \mathrm{kg} /$ day) based on clinical response to maintain phenobarbital levels in the therapeutic range. However, it corresponds with higher observed CL values in our study.

The absence of differences between weight-normalized $\mathrm{PK}$ parameters in neonates and infants indicate that maturation status contributes to the total phenobarbital distribution and elimination to a negligible part, while body size takes a major part. This is also supported by the absent dependence of the PK parameters on body size in neonates, since there were relatively small interindividual differences in body size $(2.6-4.3 \mathrm{~kg})$ in this group. However, among the infants, with a substantial body weight range $(5.2-22.0 \mathrm{~kg})$, there was a strong positive relationship between total phenobarbital distribution and elimination and body size.

As a limitation of our study no control group (nonECMO) was available. Nevertheless, we compared (Mann-Whitney test) the phenobarbital PK parameters observed in ECMO patients with our previous results in 36 critically ill neonates treated with phenobarbital, but not treated with ECMO. ${ }^{26}$ Median (IQR) phenobarbital CL and Vd in non-ECMO patients were 4.5 (3.4$5.5) \mathrm{mL} / \mathrm{h} / \mathrm{kg}$ and $0.49(0.38-0.59) \mathrm{L} / \mathrm{kg}$, respectively. The respective values for ECMO patients were 6.8 (5.4$9.8) \mathrm{mL} / \mathrm{h} / \mathrm{kg}$ and $0.46(0.34-0.75)$. Phenobarbital Vd was not significantly different $(\mathrm{p}=0.7064)$, while CL was significantly higher in ECMO patients compared to non-ECMO neonates $(\mathrm{p}=0.0001)$. Considering the 


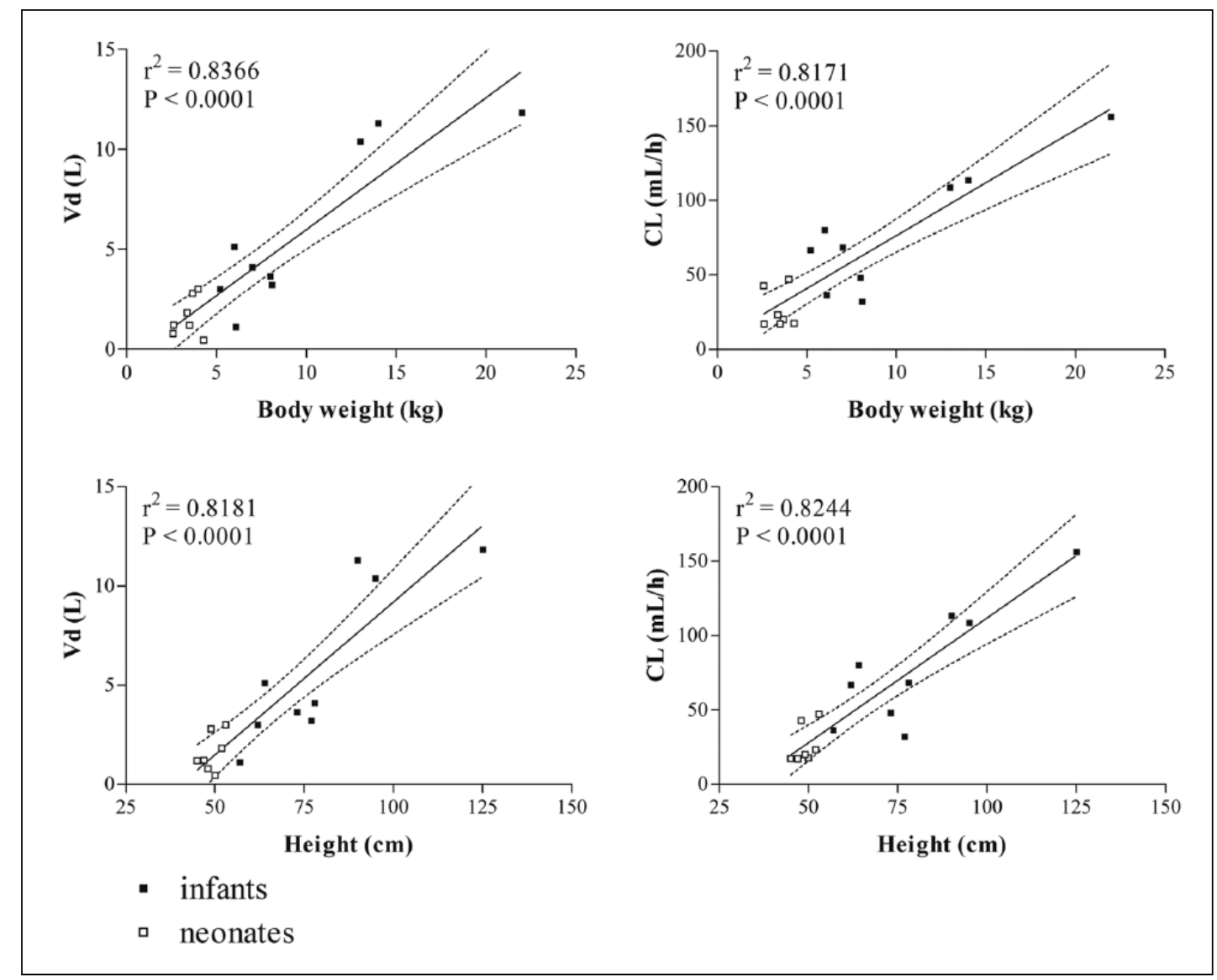

Figure 1. Relationships between phenobarbital pharmacokinetic parameters (volume of distribution $-\mathrm{Vd}$ and clearance $-\mathrm{CL}$ ) and body weight in neonates and infants during ECMO.

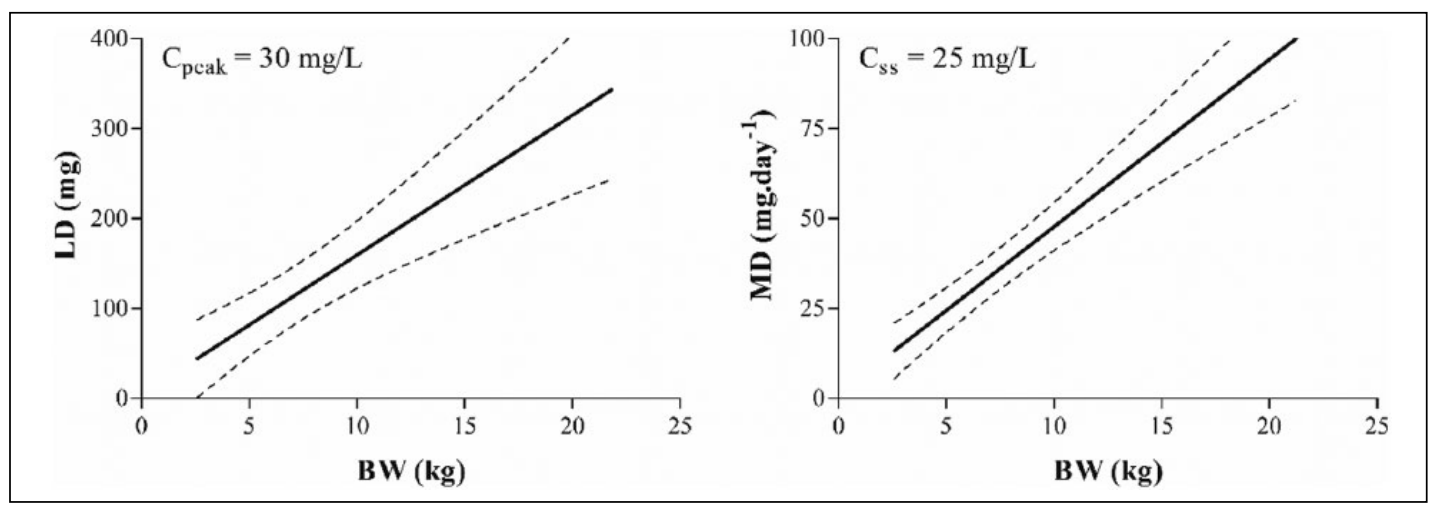

Figure 2. Nomogram for the calculation of phenobarbital loading dose (LD) and maintenance dose (MD) to reach a peak concentration $\left(C_{\text {peak }}\right)$ of $30 \mathrm{mg} / \mathrm{L}$ and steady-state concentration $\left(C_{s s}\right)$ of $25 \mathrm{mg} / \mathrm{L}$ based on body weight $(B W)$ in neonates and infants during ECMO.

same methodology in our previous study, we believe that this comparison is relevant.

Other limitations of our study are a relatively low number of patients, since it deals with a very specific cohort. However, our data are useful due to the lack of previously published data regarding phenobarbital PK in ECMO patients. Our study was based on objective PK data derived from the hospital medical records system. Therefore, the retrospective nature should not affect the validity of the results. 


\section{Conclusions}

Body size was found to be a strong PK covariate of phenobarbital disposition and, therefore, we propose dosing nomograms for both loading and daily maintenance doses of phenobarbital.

\section{Acknowledgements}

The authors are thankful to colleagues, perfusionists and nurses of the ECMO Center, General University Hospital in Prague.

\section{Declaration of Conflicting Interests}

The authors declared the following potential conflicts of interest with respect to the research, authorship and/or publication of this article: Pavla Pokorna is supported by the General University Hospital RV-project (64-165/2012) and an unrestricted research grant of the Intensive Care and Department of Pediatric Surgery of Erasmus MC-SophiaChildrens Hospital. Martin Śíma is supported by the Charles University Project Progres Q25 and a grant No. SVV 260373. The other authors do not declare any conflicts.

\section{Funding}

The authors received no financial support for the research, authorship and/or publication of this article.

\section{ORCID iDs}

Pavla Pokorná (iD https://orcid.org/0000-0001-6803-7350

Martin Šíma iD https://orcid.org/0000-0002-6541-738X

\section{References}

1. Wildschut ED, Ahsman MJ, Allegaert $\mathrm{K}$, et al. Determinants of drug absorption in different ECMO circuits. Intensive Care Med 2010; 36: 2109-2116.

2. Gill H, Thoresen M, Smit E, et al. Sedation management during therapeutic hypothermia for neonatal encephalopathy: atropine premedication for endotracheal intubation causes a prolonged increase in heart rate. Resuscitation 2014; 85: 1394-1398.

3. Wildschut ED, van Saet A, Pokorna P, et al. The impact of extracorporeal life support and hypothermia on drug disposition in critically ill infants and children. Pediatr Clin North Am 2012; 59: 1183-1204.

4. Wildschut ED, de Wildt SN, Mathot RA, et al. Effect of hypothermia and extracorporeal life support on drug disposition in neonates. Semin Fetal Neonatal Med 2013; 18 : 23-27.

5. Shekar K, Roberts JA, McDonald CI, et al. Sequestration of drugs in the circuit may lead to therapeutic failure during extracorporeal membrane oxygenation. Crit Care 2012; 16: R194.

6. Kleiber N, Mathot RAA, Ahsman MJ, et al. Population pharmacokinetics of intravenous clonidine for sedation during paediatric extracorporeal membrane oxygenation and continuous venovenous hemofiltration. $\mathrm{Br} \mathrm{J} \mathrm{Clin}$ Pharmacol 2017; 83: 1227-1239.
7. Shekar K, Roberts JA, McDonald CI, et al. Protein-bound drugs are prone to sequestration in the extracorporeal membrane oxygenation circuit: results from an ex vivo study. Crit care 2015; 19: 164.

8. Raffaeli G, Allegaert K, Koch B, et al. In vitro adsorption of analgosedative drugs in new extracorporeal membrane oxygenation circuits. Pediatr Crit Care Med 2018; doi: 10.1097/PCC.0000000000001484.

9. Schloss B, Hayes D, Jr., Tobias JD. Phenobarbital use in an infant requiring extracorporeal membrane life support. $J$ Anaesthesiol Clin Pharmacol 2013; 29: 92-94.

10. Hudak ML, Tan RC, Committee on Drugs, Committee on Fetus and Newborn, and American Academy of Pediatrics. Neonatal drug withdrawal. Pediatrics 2012; 129: e540-560.

11. Dillman NO, Messinger MM, Dinh KN, et al. Evaluation of the effects of extracorporeal membrane oxygenation on antiepileptic drug serum concentrations in pediatric patients. J Pediatr Pharmacol Ther 2017; 22: 352-357.

12. Elliott ES, Buck ML. Phenobarbital dosing and pharmacokinetics in a neonate receiving extracorporeal membrane oxygenation. Ann Pharmacother 1999; 33: 419-422.

13. Voller S, Flint RB, Stolk LM, et al. Model-based clinical dose optimization for phenobarbital in neonates: an illustration of the importance of data sharing and external validation. Eur J Pharm Sci 2017; 109S: S90S97.

14. Yukawa M, Yukawa E, Suematsu F, et al. Population pharmacokinetics of phenobarbital by mixed effect modelling using routine clinical pharmacokinetic data in Japanese neonates and infants: an update. J Clin Pharm Ther 2011; 36: 704-710.

15. Gal P, Boer HR, Toback J, Erkan NV. Phenobarbital dosing in neonates and asphyxia. Neurology. 1982; 32: 788-789.

16. Gal P, Toback J, Boer HR, Erkan NV, Wells TJ. Efficacy of phenobarbital monotherapy in treatment of neonatal seizures - relationship to blood levels. Neurology 1982; 32: 1401-1404.

17. Hall RT, Hall FK, Daily DK. High-dose phenobarbital therapy in term newborn infants with severe perinatal asphyxia: a randomized, prospective study with threeyear follow-up. J Pediatr 1998; 132: 345-348.

18. van den Broek MP, Groenendaal F, Toet MC, et al. Pharmacokinetics and clinical efficacy of phenobarbital in asphyxiated newborns treated with hypothermia: a thermopharmacological approach. Clin Pharmacokinet 2012; 51: 671-679.

19. Fischer JH, Lockman LA, Zaske D, Kriel R. Phenobarbital maintenance dose requirements in treating neonatal seizures. Neurology 1981; 31: 1042-1044.

20. Harris J, Ramelet AS, van Dijk M, et al. Clinical recommendations for pain, sedation, withdrawal and delirium assessment in critically ill infants and children: an ESPNIC position statement for healthcare professionals. Intensive Care Med 2016; 42: 972-986.

21. Finnegan LP, Connaughton JF, Jr., Kron RE, Emich JP. Neonatal abstinence syndrome: assessment and management. Addict Dis 1975; 2: 141-158. 
22. Bonett DG, Price RM. Statistical inference for a linear function of medians: confidence intervals, hypothesis testing, and sample size requirements. Psychol Methods 2002; 7: 370-383.

23. Pippenger CE, Rosen TS. Phenobarbital plasma levels in neonates. Clin Perinatol 1975; 2: 111-115.

24. Mousavi S, Levcovich B, Mojtahedzadeh M. A systematic review on pharmacokinetic changes in critically ill patients: role of extracorporeal membrane oxygenation. Daru 2011; 19: 312-321.
25. Dagan O, Klein J, Gruenwald C, et al. Preliminary studies of the effects of extracorporeal membrane oxygenator on the disposition of common pediatric drugs. Ther Drug Monit 1993; 15: 263-266.

26. Sima M, Pokorna P, Hartinger J, Slanar O. Estimation of initial phenobarbital dosing in term neonates with moderate-to-severe hypoxic ischaemic encephalopathy following perinatal asphyxia. J Clin Pharm Ther 2018; 43: 196-201. 